Research Article

\title{
Visualization of Multivariate Time-Series Characteristics of Ground Loss Caused by Shield Tunneling
}

\author{
Zhu Wen $\mathbb{D}^{1},{ }^{1}$ Xiaoli Rong $\mathbb{D},{ }^{1}$ Fei Gao $\mathbb{D}^{1},{ }^{1}$ Zhen Wang $\mathbb{D},{ }^{1}$ and Dong An ${ }^{2}$ \\ ${ }^{1}$ School of Mechanical Engineering, Nanjing University of Science and Technology, Nanjing 210094, China \\ ${ }^{2}$ Nanjing Kuntuo Civil Engineering Technology Co., Ltd., Nanjing 210000, China
}

Correspondence should be addressed to Zhen Wang; wangzhen2012@njust.edu.cn

Received 1 June 2021; Accepted 6 July 2021; Published 21 July 2021

Academic Editor: Xiao Wang

Copyright (c) 2021 Zhu Wen et al. This is an open access article distributed under the Creative Commons Attribution License, which permits unrestricted use, distribution, and reproduction in any medium, provided the original work is properly cited.

Ground loss due to earth pressure balance shield tunneling eventually leads to a surface settlement which can be an issue of great concern. However, the existing machine learning methods ignore the continuous and dynamic nature of EPB shield tunneling. In this work, a multivariate time-series (MTS) model for ground loss is proposed based on an analysis of factors and processes related to ground loss combined with the characteristics of original time-series data involving multiple parameters recorded by EPB shield machines in real time. A method of visualizing MTS features based on a residual network and multichannel fully convolutional neural network is also presented. The validity of the proposed ground-loss model is verified via calculation and comparison with 13 EPB shield construction projects carried out in typical urban areas featuring soft soil. Thermal maps are thus obtained to visualize the classification contributions, which provide a visual basis for feature analysis.

\section{Introduction}

Earth pressure balance (EPB) shield tunneling has become one of the main methods used to construct urban metro lines. This is because of its high construction efficiency, wide range of applications, and limited impact on the ground surface and associated human activity. However, EPB shield construction will inevitably disturb the surrounding soil causing, to some extent, ground loss and settlement of the ground surface [1-11]. In particular, using the method to construct shallowly buried tunnels in typical urban areas with soft soil poses a serious threat to structures both on the ground surface and underground [12, 13]. Many scholars have attempted to estimate ground loss due to EPB shield tunneling using empirical methods [1-3], theoretical methods [4-6], and numerical simulation [7-10]. However, the complex engineering situation limits the practical application effect of these methods. It is worth noting that the construction process data with rich feedback of tunneling state are automatically recorded during the tunneling process of the earth pressure balance shield machine, including the important information of controlling the tunneling process of the earth pressure balance shield machine, which provides the data basis for the research of related engineering problems based on the machine learning method $[14,15]$. And the success of artificial intelligence technology in computer vision and natural language processing also provides a reference for data-based prediction methods.

Kim et al. [16], for example, used 113 datasets from the construction of the Seoul Metro and applied an artificial neural network (ANN) to predict surface settlement and analyze the relative importance of various influencing factors. By considering three factors affecting surface settlement (geological conditions, tunnel geometry, and shield operation), Suwansawat and Einstein [17] used 49 datasets from a tunnel project in Bangkok to predict the surface settlement caused by EPB shield tunneling. These authors also used an ANN and found that there is an internal relationship between the influencing factors. Darabi et al. [18] used empirical, numerical, and neural network methods to analyze the subsidence and convergence of the tunnel in Tehran No. 3 subway line, and through comparison, they suggested utilizing the ANN to estimate the tunnel subsidence. Ninić and Meschke [19] proposed a method for the simulation 
supported steering of the mechanized tunneling process in real time during construction and used Particle Swarm Optimization (PSO) to optimize ANN for the inverse analysis; i.e., identification of model parameters according to monitoring results obtained during tunnel advance allows the update of the model to the actual geological conditions in real time. Zhang et al. [20-22] took the response of the surface to shield tunneling to be a complex problem affected by the tunnel geometry, operating parameters of the shield machine, geological conditions, and any abnormal conditions. They analyzed 236 datasets taken from the construction of Metro Line 4 in Changsha City in their work. They proposed two novel computational models based on the random forest (RF) algorithm to support the automatic steering of the EPB shield tunneling machine. PSO-RF was used to optimize the operating parameters of the shield machine when the settlement exceeded the maximum allowed value. In addition, Deep-Q network PSO was used to optimize the weight and deviation of the extreme learning machine and the relationships between the influencing factors and ground response were identified through engineering practice.

In addition to the above methods, approaches based on relevance vector machines [23], adaptive neurofuzzy inference systems [24], genetic expression programming [25], least-squares support vector machines [26], and general regression neural networks [27] have also been used to predict settlement. Many scholars have tried to use ML to predict the ground settlement based on the abundant amount of EPB shield tunneling data that is available, as shown in Figure 1. In each case, the surface settlement due to ground loss is assumed to be related to many factors, e.g., how the shield is operated and geological conditions. However, ground loss due to EPB tunneling is a continuous and dynamic process. Therefore, the original data in the EPB construction log actually consists of a collection of time series. The temporal and spatial characteristics of the EPB shield tunneling process are ignored in the existing research and the selection of datasets lacks knowledge of the exact conditions and processes involved. Moreover, the amount of data available for a particular project is generally small and so there is a risk that the calculations suffer from overfitting. Therefore, the use of a supervised multivariate time series (MTS) model is proposed in this work that is based on an indepth analysis of the continuous and dynamic ground loss due to EPB shield tunneling combined with the time series characteristics of the original data.

As the model lacks knowledge of the domain, it is impossible to select features manually. However, the end-toend deep-learning method used eliminates the deviation caused by manual design features and also learns the most discriminating and useful features for classifying tasks [28, 29]. Wang et al. compared nine deep-learning algorithms using 12 MTS datasets [30-32]. They found that residual networks (ResNets) and fully convolutional neural networks (FCNs) produce the best results. Furthermore, ResNets have better stability. In terms of MTS classification, one-dimensional (1D) convolution combined with gradientweighted class activation mapping (Grad-CAM) can be used to facilitate feature visualization along the time axis $[33,34]$. However, there is no basis for the ranking of multivariate parameters and convolution along the directions of parameters is meaningless. As a result, the relative importance of the relationships between multivariate parameters cannot be analyzed. Therefore, in this paper, we construct a method that combines a ResNet with a multichannel fully convolutional neural network (MCFCN) based on techniques developed for multichannel deep convolutional neural networks (MCDCNNs) $[35,36]$. The new method allows feature visualization to be realized along the time axis and along parameter directions using Grad-CAM. It also learns which features are related to ground loss by highlighting subsequences that contribute most to the classification.

An MTS model for ground loss is also constructed in this work based on $13 \mathrm{EPB}$ shield tunneling projects carried out in soft soil typically encountered in urban parts of Changzhou in China. Classification contribution thermal maps are subsequently calculated based on the effective classification provided by the ResNet and MCFCN. In addition, the relevant characteristics are analyzed. The rest of this paper is organized as follows. The MTS model for ground loss due to EPB shield tunneling is established in Section 2 and the ResNet-MCFCN visualization method is presented in Section 3. The experiments and analysis of the corresponding experimental results are presented in Section 4. Finally, the conclusions of the paper are given in Section 5.

\section{MTS Ground Loss Model}

Ground loss due to EPB shield tunneling is a continuous and dynamic process. Therefore, the construction data recorded in real time from an EPB shield machine has clear time-space correspondence. Considering the continuous and dynamic process and establishing a reasonable model is the key to studying ground loss using ML.

2.1. Volume Loss. As shown in Figure 2, the construction of each supporting segment is taken as the basic unit in the EPB shield tunneling process. There are two main stages: shield advance and segment installation. The process of tunnel engineering is realized by the construction of repeated supporting segments. The ground loss during EPB shield tunneling eventually leads to surface settlement.

Peck [1] first proposed the concept of land subsidence troughs with quasinormal distributions based on an analysis of a large amount of land subsidence data and associated engineering data [1]. Under the assumption that the soil is undrained and incompressible, the surface settlement due to ground loss is considered to be given by the following:

$$
V_{\text {loss }}=\frac{\sqrt{2 \pi}}{2}\left(\frac{H}{D}\right)^{n} D S_{\max }
$$

where $V_{\text {loss }}$ is the volume loss (i.e., the volume between the original and settled ground surfaces per meter of tunnel advance), and as shown in Figure $1, S_{\max }$ is the maximum surface settlement above the tunnel axis, $D$ is the diameter of the shield-driven tunnel, and $H$ is the depth of the tunnel 


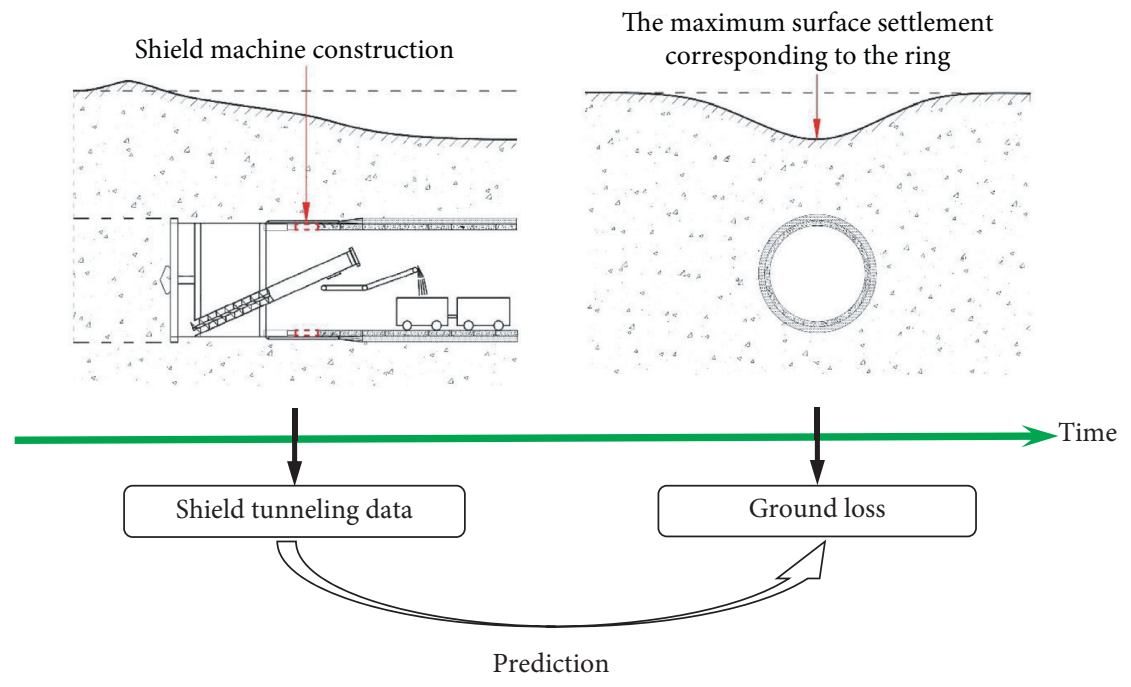

Figure 1: Prediction based on shield construction data.

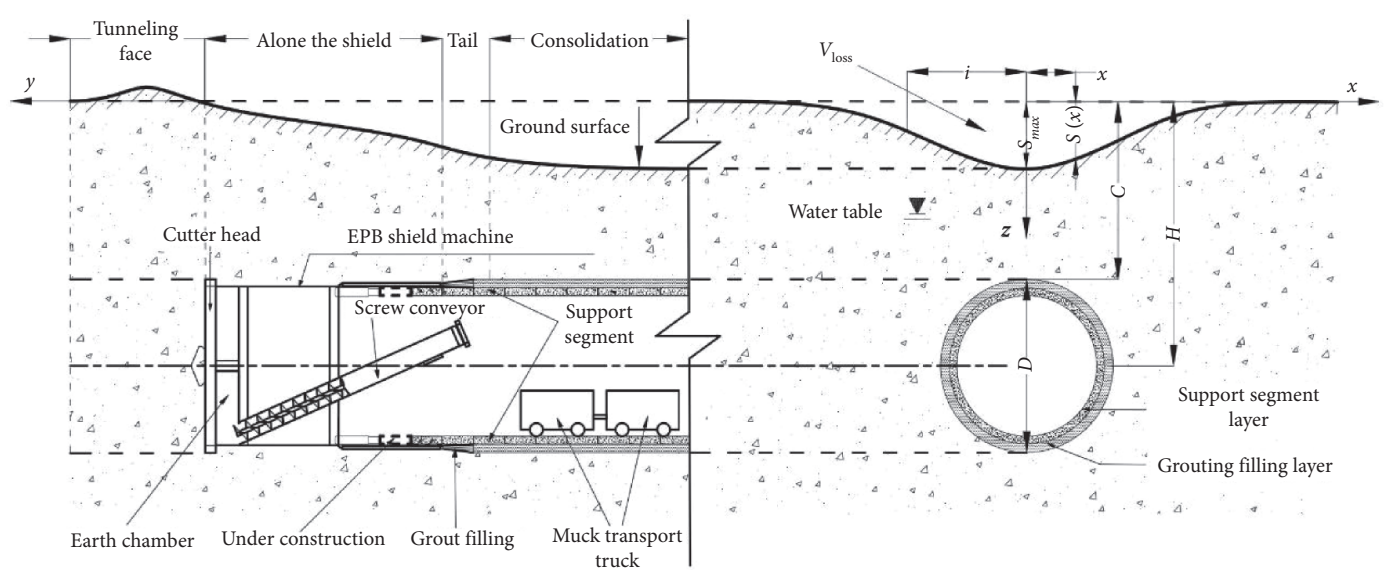

Figure 2: Ground loss due to EPB shield tunneling.

axis with respect to the original ground surface. The index in equation (1), $n$, lies in the range $0.8-1.0$, larger values corresponding to softer soil. For soft soil, suppose $n \approx 1$ :

$$
V_{\mathrm{loss}}=\frac{\sqrt{2 \pi}}{2} H S_{\max }
$$

Thus, equation (2) implies that $V_{\text {loss }} \propto H S_{\max }$. Sagaseta analyzed the strain field arising in incompressible soil due to ground loss at a certain depth below the ground and used absolute displacement as a variable to derive the displacement and stress fields in the soil below the ground [4]. The results of this analysis also suggested that $V_{\text {loss }} \propto \mathrm{HS}_{\max }$.

As mentioned in the introduction, many studies target $S_{\max }$ as a means of predicting surface settlement. However, for a given set of geological conditions, the surface settlement due to ground loss is directly related to the shield diameter $(D)$ and burial depth of the tunnel $(C)$. These two scale factors are related to the depth $(H)$ of the tunnel axis below the ground surface. In addition, as indicated by equation (2), a large amount of engineering experience shows that the product of $S \max$ and $H$ is representative of the unit ground loss area in the tunneling direction which can be used to characterize the ground loss due to the EPB shield tunneling process. Therefore, for given geological conditions, it is more reasonable to use the ground loss to measure the response of the ground to EPB shield tunneling than surface settlement. However, because the surface settlement is more intuitive as the variable monitored, the equivalent average final settlement, $\bar{S}_{\max }$, can be obtained by dividing the ground loss $V_{\text {loss }}$ of all sample points by the average depth of the tunnel axis beneath the ground, $\bar{H}$.

2.2. Influence Factors. An EPB shield machine uses the continuous flow of modified excavation material as a support medium. The flow of excavation material through the cutter head, soil chamber, and screw conveyor is carefully controlled (by adjusting parameters such as thrust, speed of advance, cutter head speed, and speed of the screw conveyor) $t+o$ establish a dynamic pressure balance. In this way, the stability of the tunnel face is assured and the shield machine tunnels smoothly through the ground. As shown in Figure 2, the region in which ground loss occurs can be 
divided into four main parts: tunneling face, alone the shield, tail, and consolidation according to the relative position of the shield and surface monitoring points [11]. The dynamic interaction between shield machine and surrounding soil and the continuous response of stratum are the main causes of ground loss. Combined with the process of dynamic interaction between shield machine and soil, through analysis, the five main influencing factors of ground loss caused by shield tunneling are further summarized, as shown in Figure 3.

(1) Interaction between cutter head and soil: some ground is lost in front of the cutter head of the EPB shield machine as it cuts and squeezes the soil [37-40]. The EPB shield machine cuts the soil according to the speed and torque of the cutter head. This action is combined with the pressure and speed of advance of the machine to squeeze the soil in front of the cutter head, as shown in Figure 3.

(2) Balance between the amount of advance and discharge: the advancing capacity of the EPB shield machine is the product of the cutter head area and advancing speed and the discharge volume is the conveying capacity of the screw conveyor. These two quantities are roughly balanced on the whole [41-43]. However, when the advance rate is greater than the discharge volume, the pressure in the soil chamber increases and the shield has a squeezing effect on the soil. When the converse is true, the pressure in the soil chamber decreases, and there is an increased risk of ground loss.

(3) Direction of advance and attitude: some ground loss occurs radially as the soil around the shield enters the gap between the shield and the surrounding soil. The loss also occurs when the soil is squeezed and irreversibly deformed by the shield due to a change in the direction of advance or attitude of the shield, such as snake-like motion and large angle turning [44, 45].

(4) Grouting of the tail: as the shield advances, an annular cavity is formed between the segment pushed out of the shield and the surrounding soil. Therefore, grouting is synchronously applied at the tail of the shield to protect the segment and prevent the surrounding soil from entering the gaps. Besides filling the annular cavity, controlling the amount of grout used and the pressure used to apply it allows some of the ground loss at the tunneling face and shield-only region to be compensated for [46].

(5) Consolidation settlement: after the support segments are installed, slurry shrinkage and stress changes in the strata may lead to long-term consolidation settlement of the soil above the tunnel [47]. However, this only makes a very small contribution to ground loss and so this factor is ignored in the rest of this paper.

According to the above five influence factors, the relevant parameters of shield construction data are summarized as shown in Table 1 . The shield tunneling data mainly includes the cutter head torque, the cutter head speed and pushing speed of the feedback cutter head state, the pushing displacement and pushing pressure parameters of the feedback pushing state, the screw conveyor torque and speed parameters of the feedback screw conveyor in the discharged state, the grouting pressure and grouting amount parameters of the feedback shield tail grouting state, and the earth chamber pressure parameters of the feedback earth pressure, as well as the feedback of the shield tunneling attitude of horizontal/vertical difference between the front and rear points.

Shield construction data is a typical time series data, according to the construction stage can be divided into shield tunneling stage and segment assembly stage. Take the cutter head torque, pushing displacement, and screw conveyor torque of a shield tunnel construction process as an example, as shown in Figure 4. From the data of shield construction, we can also observe the process of repeated driving stage and assembling segment of the ring. The interaction between shield machine and soil affects the gr+ound loss mainly in the shield tunneling stage.

As the dynamic interaction between shield machine and soil mainly occurs in the shield tunneling stage, the shield machine stops tunneling in the assembly stage. Therefore, the shield construction data considered in this paper is the corresponding part of the shield tunneling stage. Taking the cutter head torque, pushing displacement, and screw conveyor torque in the shield tunneling process as an example, the multiring construction process is considered, as shown in Figure 5.

Ground loss due to EPB shield tunneling is not only a dynamic process that depends on many factors but also a process that varies continuously in time and space. As shown in Figure 6, the length of the EPB shield machine, $L_{m}$, is usually much greater than the width of the supporting segments, $l$. The burial depth of the tunnel, $C$, is also usually much greater than the diameter of the shield machine, $D$. Therefore, the continuity of the ground determines that the settlement at the surface is related to the construction of several rings of supporting segments. In addition, when $L_{m}$ is larger than the distance between the monitoring points on the surface, there is a correlation between the observations made at the front and rear monitoring points.

As shown in Figure 6, the settlement of the current settlement observation point starts at ring S-7 of the tunnel when the cutter head of the machine advances from ring S-3 to $S-2$. At this time, the support segment corresponding to ring $\mathrm{S}-9$ is being pushed out of the EPB shield machine and simultaneously grouted. During the construction process, data (e.g., cutter head torque, speed of advance, grouting pressure, etc.) is synchronously recorded in real time at the monitoring point corresponding to ring S-7. Each parameter $X$ is recorded in the form of a univariate time series $X=\left[x_{1}, x_{2}, \ldots, x_{t}\right]$, where $x_{t}$ is the data point and $t$ is the length of the time series. Therefore, the ground loss caused by EPB shield tunneling is treated as a dynamic and continuous process and has a clear time-space correspondence. 


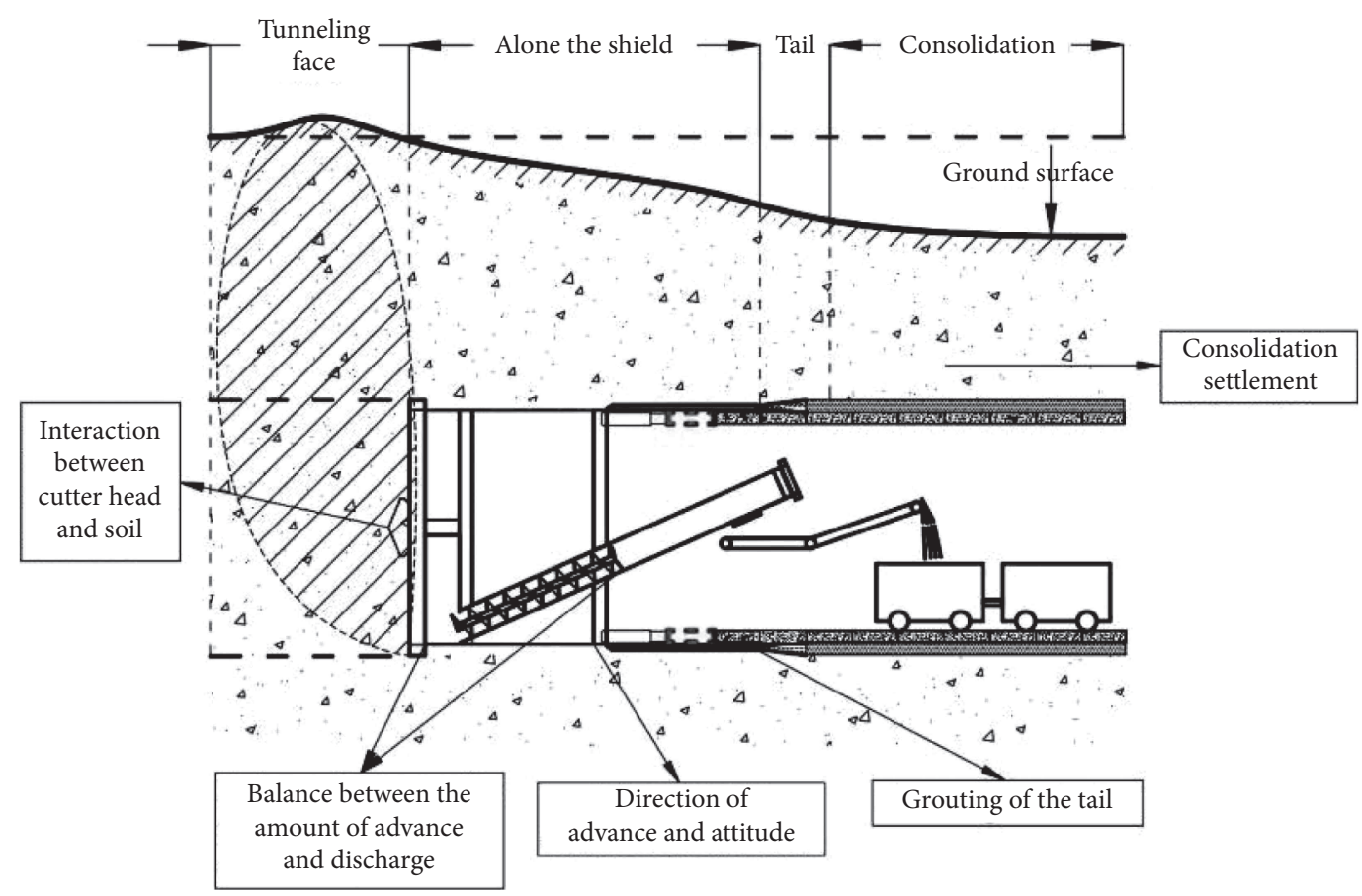

FIgURE 3: Five main factors affecting the ground loss resulting from EPB shield tunneling.

TABLE 1: Shield construction parameters related to ground loss.

\begin{tabular}{|c|c|c|c|c|}
\hline & Parameter & Unit & Function & Related influencing factors \\
\hline 1 & Thrust pressure: up & Bar & \multirow{5}{*}{$\begin{array}{l}\text { Control the direction of advance and } \\
\text { feedback the squeezing effect on the soil }\end{array}$} & \multirow{5}{*}{$\begin{array}{l}\text { Interaction between the cutter head and soil; } \\
\text { direction and attitude }\end{array}$} \\
\hline 2 & Thrust pressure: down & Bar & & \\
\hline 3 & Thrust pressure: left & Bar & & \\
\hline 4 & Thrust pressure: right & Bar & & \\
\hline 5 & Earth chamber pressure: up & Bar & & \\
\hline 6 & $\begin{array}{c}\text { Earth chamber pressure: } \\
\text { medium }\end{array}$ & Bar & \multirow[t]{2}{*}{$\begin{array}{l}\text { Feedback the earth pressure distribution } \\
\text { and squeezing effect on the soil }\end{array}$} & \multirow[t]{2}{*}{$\begin{array}{l}\text { Interaction between the cutter head and soil; } \\
\text { balance between advance and discharge }\end{array}$} \\
\hline 7 & Earth chamber pressure: down & Bar & & \\
\hline $\begin{array}{l}8 \\
9\end{array}$ & $\begin{array}{l}\text { Torque of the cutter head } \\
\text { Speed of the cutter head }\end{array}$ & $\begin{array}{c}\mathrm{kNm} \\
\mathrm{rpm}\end{array}$ & \multirow{2}{*}{$\begin{array}{l}\text { Control the cutting speed of soil and } \\
\text { feedback its degree of hardness }\end{array}$} & \multirow[t]{2}{*}{ Interaction between the cutter head and soil } \\
\hline 10 & & $\mathrm{~mm} /$ & & \\
\hline 10 & Advance pushing speed & $\min$ & \multirow{3}{*}{$\begin{array}{c}\text { Control the speed of advance and quantity } \\
\text { of shield constructed }\end{array}$} & \multirow{3}{*}{ Balance between advance and discharge } \\
\hline 11 & $\begin{array}{c}\text { Average pushing displacement } \\
\text { Average articulation }\end{array}$ & $\mathrm{mm}$ & & \\
\hline 12 & displacement & $\mathrm{mm}$ & & \\
\hline 13 & $\begin{array}{l}\text { Horizontal difference between } \\
\text { the front and rear points }\end{array}$ & & \multirow{2}{*}{$\begin{array}{l}\text { Feedback on the direction and attitude of } \\
\text { the tunneling process }\end{array}$} & \multirow[b]{2}{*}{ Direction and attitude } \\
\hline 14 & $\begin{array}{l}\text { Vertical difference between the } \\
\text { front and rear points }\end{array}$ & & & \\
\hline $\begin{array}{l}15 \\
16\end{array}$ & $\begin{array}{l}\text { Torque of the screw conveyor } \\
\text { Speed of the screw conveyor }\end{array}$ & $\begin{array}{c}\mathrm{kNm} \\
\mathrm{rpm}\end{array}$ & $\begin{array}{l}\text { Control the rate of discharge from the } \\
\text { shield machine }\end{array}$ & Balance between advance and discharge \\
\hline 17 & Total amount of grouting & $\mathrm{L}$ & Control the quantity of grout used and & \multirow{2}{*}{ Tail grouting } \\
\hline 18 & Average grouting pressure & bar & feedback grouting pressure & \\
\hline
\end{tabular}

As a result, it is necessary to consider not only the processes involved but also the characteristics of the original data in the MTS model.

2.3. MTS Model. Due to the dynamic and continuous nature of the ground loss process, the characteristics of the original data from the shield construction process are complex multivariate time series. In the absence of experience to guide feature selection, we consider all the time series data of relevant parameters in a unified way. We thus propose a supervised MTS model consisting of multiparameter time series connected via the data sequence to the multiring construction process as data $X_{N}$. The corresponding ground loss is expressed as $Y_{N}$, as shown in Figure 7. Assuming the 


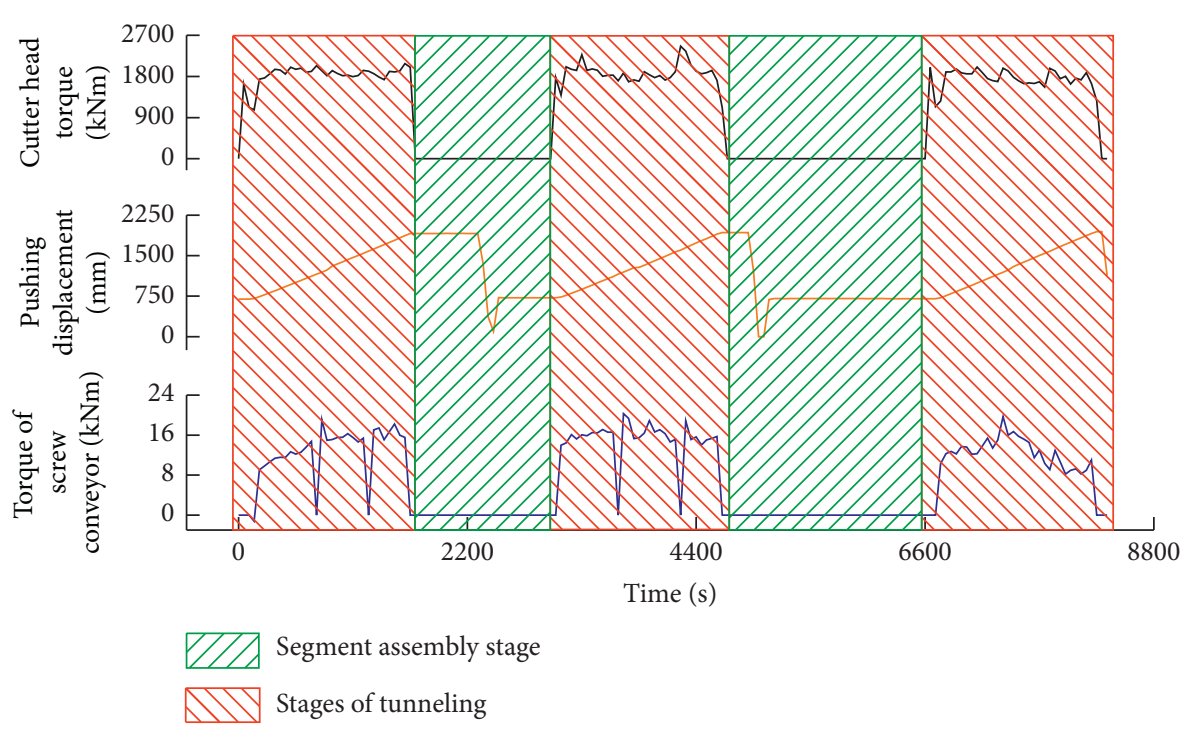

FIgURE 4: Shield construction data of two-stage circulation.

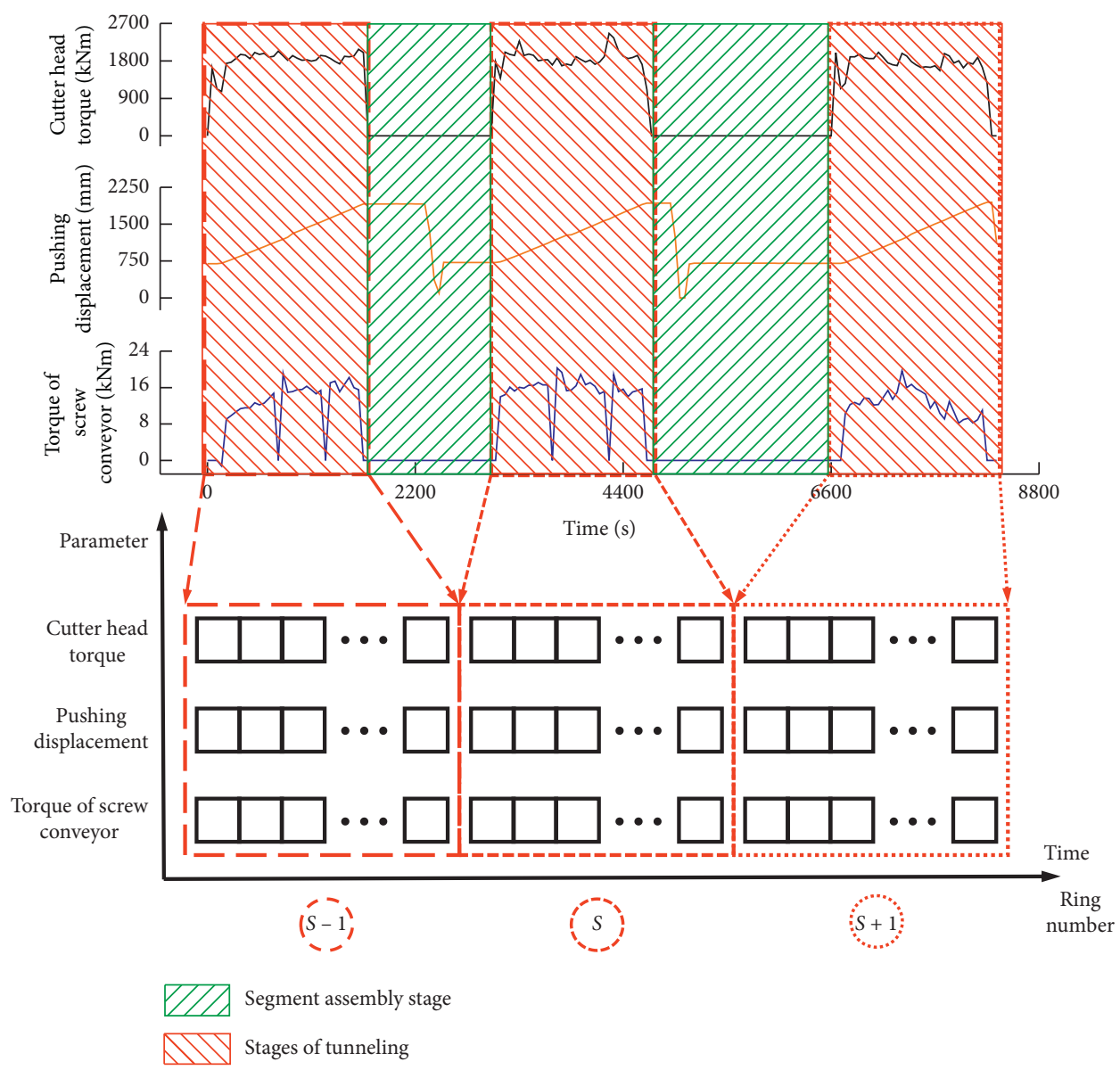

FIGURE 5: Multiring data of shield tunneling stage.

geological conditions are similar throughout, the MTS model for the ground loss due to EPB shield tunneling can be divided into the following four steps:
(1) The data in $X_{N}$ starts at ring $S-b$. It can be seen from Figure 6 that the effect of the shield machine on the ground loss at the monitoring point essentially 


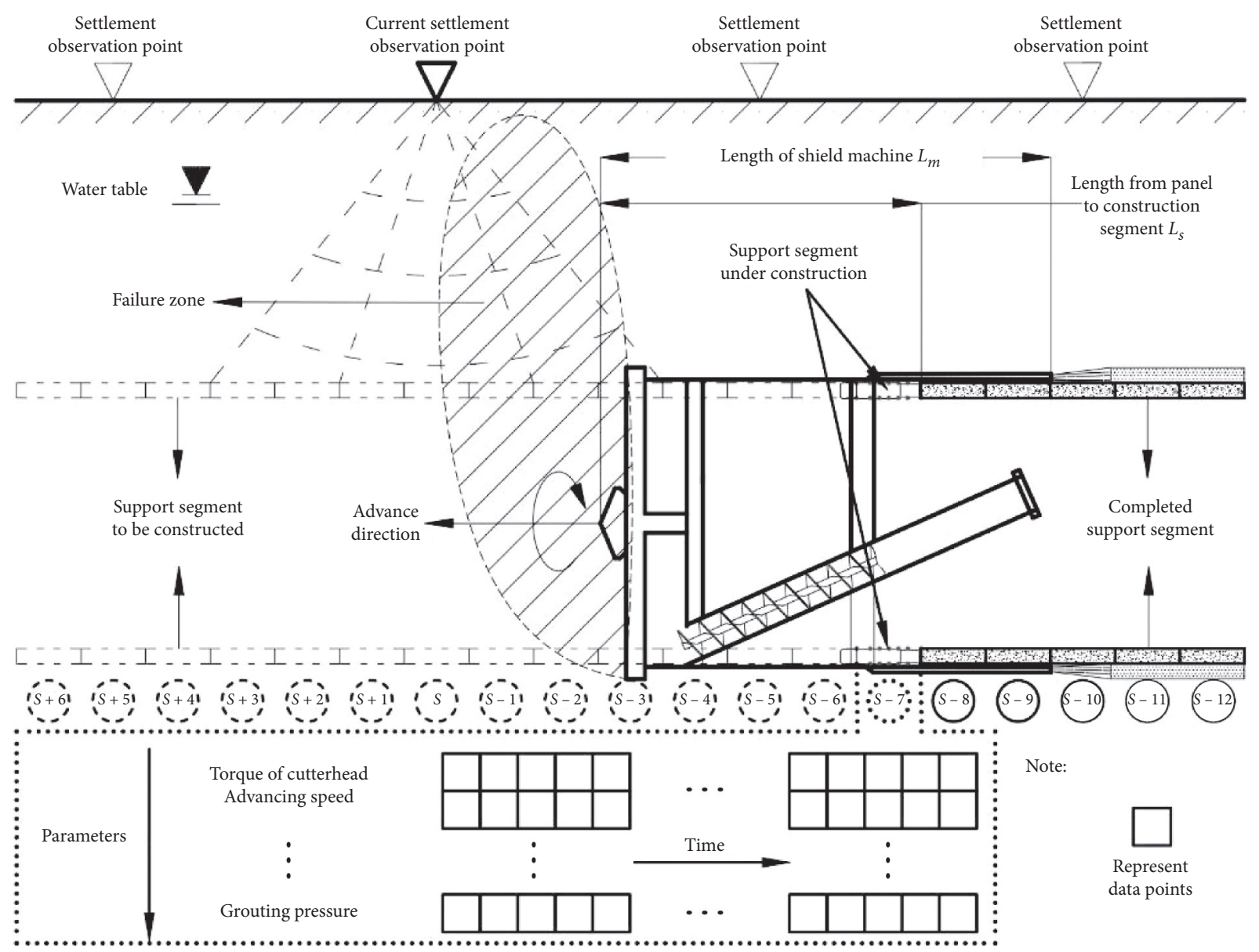

FIgURE 6: Schematic diagram showing the dynamic and continuous process of ground loss due to EPB shield tunneling.

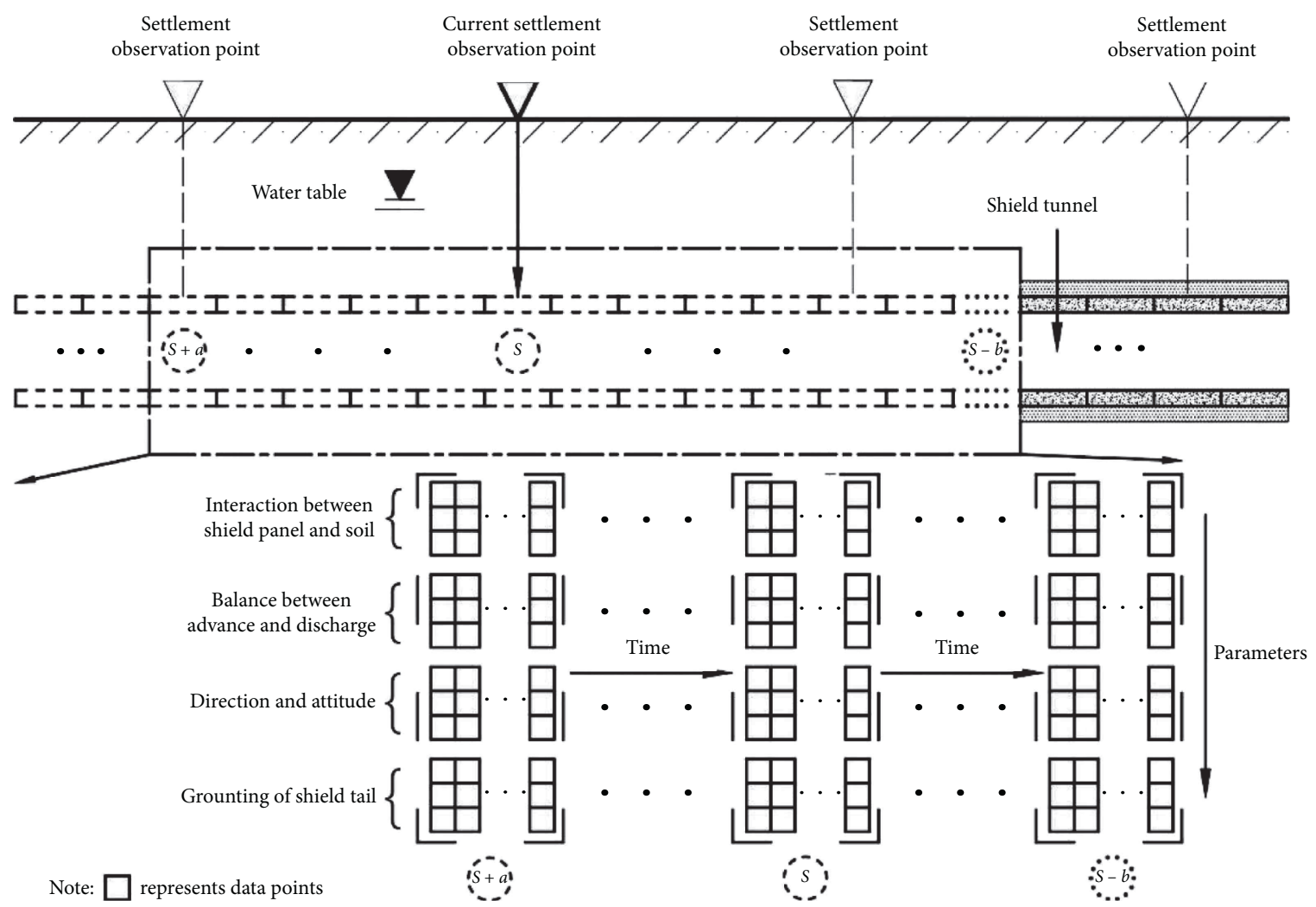

FIgURE 7: The MTS model for ground loss. 
starts when the squeezing effect of the shield machine forms a failure zone. The failure zone is usually similar to a chimney and depends on the diameter of the EPB shield machine $(D)$, the burial depth of the tunnel $(C)$, and the geological conditions. It is assumed that the failure zone is the main area affecting the ground monitoring points. It has been reported by Zhang et al. [39] that the boundary of the failure zone is about $0.33 \mathrm{D}$ in front of the tunnel face. Considering the nature of the project case, $C / D>1$ in this paper, so the range of influence is increased to $0.5 \mathrm{D}$ in front of the tunnel. Therefore, as shown in Figure 7, the influence of the shield machine on the surface settlement measuring point corresponding to ring $S$ starts from the construction of ring $S-b$, where

$$
b=\left\lfloor\frac{\left(0.5 D+L_{s}\right)}{l}\right\rfloor .
$$

in which $L_{s}$ is the distance between the panel and support section under construction and \lfloor\rfloor represents the integer operation.

(2) The data in $X_{N}$ ends at $S+a$. As shown in Figure 6, the grouting position at the tail of the shield is usually $L_{m}-\mathrm{L}_{\mathrm{s}}$ after the segment supported by the current construction ring. The grouting quantity and grouting pressure are regarded as the key factors controlling surface settlement. However, research on the scope of the influence of the shield tail grouting process has not yet been reported. Therefore, we assume that the scope of this influence on the ground surface is the same as that of the shield compaction effect. Hence, the influence of the shield machine on the surface settlement measuring point corresponding to ring $S$ ends at the construction of ring $S+a$, as shown in Figure 7, where

$$
a=\left\lfloor\frac{\left(0.5 D+L_{m}-L_{s}\right)}{l}\right\rfloor,
$$

and $L_{m}$ is the total length of the shield machine.

(3) Related parameters: according to the four main factors mentioned in Section 2.2, the relevant parameters are (1) the interaction between the cutter head and soil (thrust pressure, soil chamber pressure, cutter head speed, cutter head torque, etc.), (2) the balance between advance and discharge (advance speed, advance displacement, soil chamber pressure, screw conveyor speed, screw conveyor torque, etc.), (3) the direction and attitude (horizontal and vertical deviation between the specific front and rear points), and (4) tail grouting (total amount and average grouting pressure used in the shield tail). The data are obtained in the form of an MTS by combining the time series data for the relevant parameters, where $X^{K}$ is the univariate time series for parameter $K$.

(4) According to the discussion in Section 2.1, under similar geological conditions, the $\mathrm{HS}_{\max }$ value at a monitoring point can be used to represent the degree of ground loss, i.e., as $Y_{N}$.

Finally, under the assumption that the ground conditions are similar throughout, a supervised MTS model $D T=\left\{\left(X_{1}, Y_{1}\right),\left(X_{2}, Y_{2}\right), \ldots,\left(X_{N}, Y_{N}\right)\right\}$ is formed, which includes the interaction between the cutter head and soil, the balance between shield advance and discharge, the direction and attitude of the shield, and tail grouting. The product of the maximum surface settlement $\left(S_{\max }\right)$ at the corresponding position and the depth $(H)$ from the tunnel axis to the ground is $Y_{N}$. According to previous engineering experience, the surface settlement can be divided into three cases: (i) surface uplift, (ii) normal settlement, and (iii) excessive settlement. If the quantity of data is large enough, it can be further divided. At the same time, it can be seen from Section 2.1 that the ground loss is the surface settlement, and so the ground loss can also be divided according to the surface settlement.

\section{Visualization Method}

There are five kinds of typical discriminatory characteristics found in univariate time series, referred to as whole series, intervals, shapelets, dictionary based, and spectral [48]. The MTS contains characteristic variables and the relationship between them is more complex. Due to the lack of knowledge of the domain in the MTS model, we use a method that combines ResNets with MCFCNs. Furthermore, Grad-CAM is used to visualize the data along the time axis and parameter directions. By highlighting the subsequences that contribute the most to a certain classification, we can learn the characteristics associated with the ground loss.

3.1. Time Axis Direction. The MTS sequence along the time direction is known, so $1 \mathrm{D}$ convolution can be performed along the time axis to extract features. However, the order of the parameters is uncertain. As shown in Figure 8, the basic $1 \mathrm{D}$ convolution module is a $1 \mathrm{D}$ convolution layer connected to a batch normalization layer [49] and a ReLU activation layer. The $1 \mathrm{D}$ convolution is filled without striding. Overall, the basic $1 \mathrm{D}$ convolution module $\operatorname{Conv} B_{k}^{f}$ can be expressed via the equations:

$$
\begin{aligned}
\mathbf{y}_{k} & =\boldsymbol{\omega}_{k} \otimes \mathbf{x}+\mathbf{b}_{k}, \\
\widehat{\mathbf{y}}_{k}^{f} & =\operatorname{Conv1D}\left(\mathbf{y}_{k}\right), \\
\mathbf{h}_{k}^{f} & =\operatorname{ReLU}\left(B N\left(\widehat{\mathbf{y}}_{k}^{f}\right)\right), \\
\mathbf{h}^{f} & =\operatorname{ConvB}_{k \curvearrowleft 88,5,3\}}^{f}(\mathbf{x}),
\end{aligned}
$$

where $\otimes$ represents the convolution operator, Conv1D $f$ denotes 1D convolution using $f$ filters, and batch normalization $(\mathrm{BN})$ is applied to speed up the rate of convergence and help improve generalization. Each convolution operation consists of three basic modules with kernels sizes $k=\{8,5,3\}$. This structure has proved to be the best one to use in many MTS classification tasks [30, 31]. Using a ResNet 


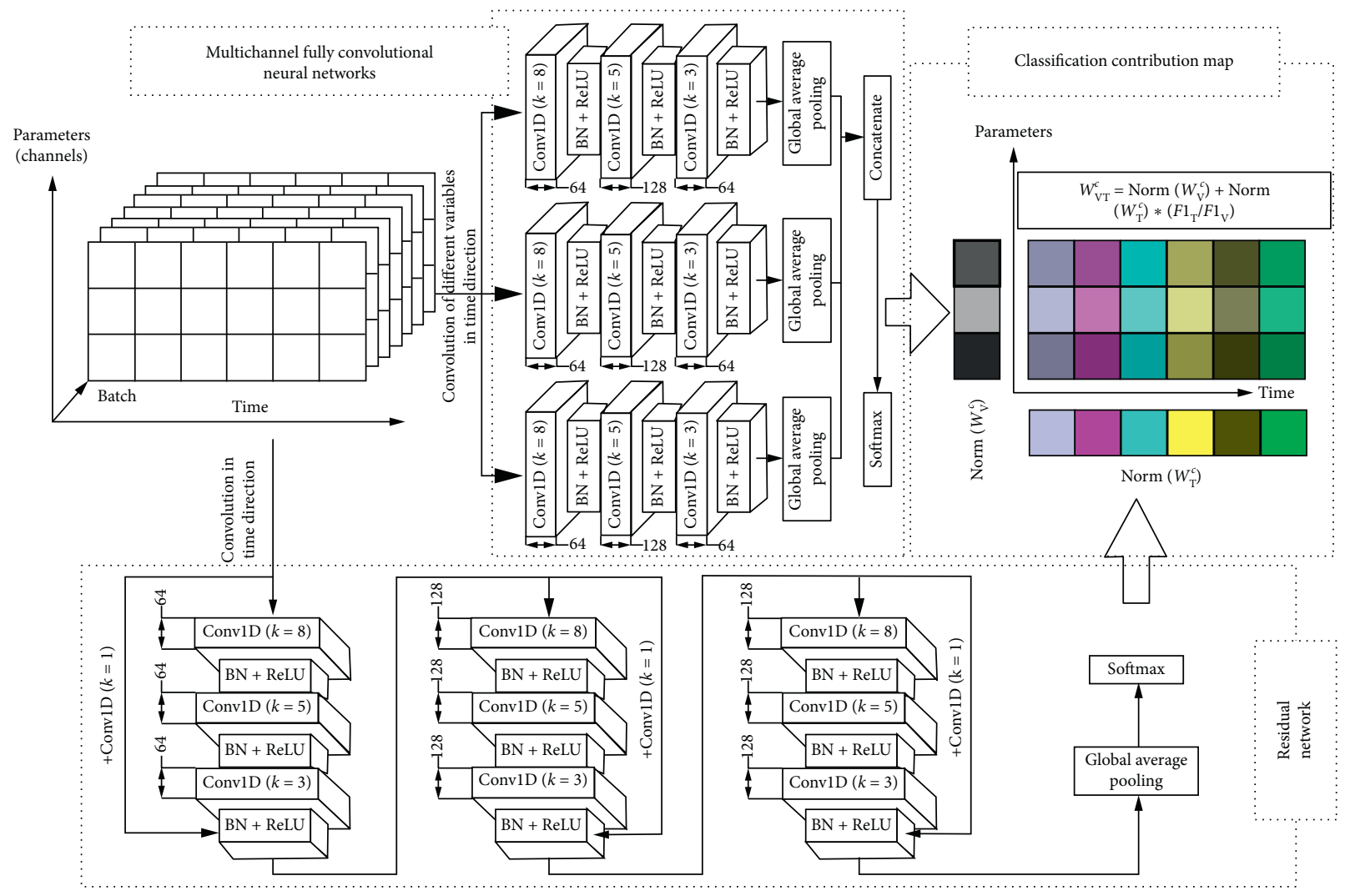

FIgURE 8: method used to visualize the classification features of the MTS.

is equivalent to adding a linear shortcut to the basis of the convolution. It connects the output of the residual block to its input so that the gradient flow can directly pass through the connections made. This makes it easier to train the deep neural network (DNN) by reducing the vanishing gradient effect [50]. As shown in Figure 8, the residual module can be expressed via the following equations:

$$
\begin{aligned}
& \mathbf{h}_{1}=B_{f 1}(\mathbf{x})=\operatorname{ReLU}\left(B N\left(\mathbf{h}^{f 1}\right)+\mathbf{x}\right), \\
& \mathbf{h}_{2}=B_{f 2}\left(\mathbf{h}_{1}\right)=\operatorname{ReLU}\left(B N\left(\mathbf{h}^{f 2}\right)+\mathbf{h}_{1}\right), \\
& \mathbf{h}_{3}=B_{f 3}\left(\mathbf{h}_{2}\right)=\operatorname{ReLU}\left(B N\left(\mathbf{h}^{f 3}\right)+\mathbf{h}_{2}\right),
\end{aligned}
$$

where $B_{f i}$ denotes a convolutional block using $f i$ filters $(f 1=64 ; f 2=f 3=128)$. The final ResNet stacks three residual blocks, followed by a global average pooling (GAP) layer and a softmax layer. In order to increase the interpretability of the DNN, reveal the reliability of the classifier and accumulate the experience and knowledge related to classification, CAM is introduced [33].

Suppose that the classification score of class $c$ is $S_{c}$, the weight of the GAP operator is $\omega_{i}^{c}$, the size of the eigenvectors is $t=1 * t$, the $i$ th eigenvector is $F_{i}$, the eigenvalue of $j$ position of $i$ eigenvector is $F_{i}^{j}$, and the number of filters in the last layer is $I$, then,

$$
\begin{aligned}
S_{c} & =\sum_{i=1}^{I} \omega_{i}^{c} \operatorname{GAP}\left(F_{i}\right) \\
& =\sum_{i=1}^{I} \omega_{i}^{c} \frac{1}{t} \sum_{j=1}^{t} F_{i}^{j} \\
& =\frac{1}{t} \sum_{i=1}^{I} \sum_{j=1}^{t} \omega_{i}^{c} F_{i}^{j},
\end{aligned}
$$

Equation (7) shows that the greater the value of $\omega_{i}^{c}$, the greater the contribution made by $F_{i}$ to the classification, and the more important it is to the classification. This allows a credible visual interpretation to be made for the model to help decision-making, which is very important for accumulating experience related to classification. Grad-CAM is an equivalent method to CAM [34] that is capable of visualizing classification contributions without using a GAP layer. The Grad-CAM $W_{T}^{c}$ along the time axis satisfies the following conditions:

$$
\begin{aligned}
\alpha_{i}^{c} & =\frac{1}{t} \sum_{j=1}^{t} \frac{\partial S_{c}}{\partial F_{i}^{j}}, \\
W_{\mathrm{T}}^{c} & =\operatorname{ReLU}\left(\sum_{i} \alpha_{i}^{c} F_{i}\right) .
\end{aligned}
$$


3.2. Parameter Direction. A ResNet combined with GradCAM allows the classification contributions of the data points to be visualized along the time axis but cannot visualize the classification contributions between multiple parameters. Therefore, an MCFCN is constructed, as shown in Figure 8.

Each characteristic variable is taken as a channel and convolution modules similar to those shown in equation (5) are set up. Each convolution operation includes three basic modules with kernels sizes $k=\{8,5,3\}$. The basic module of the MCFCN, $M C_{n}$, can be expressed in the form:

$$
\mathrm{MC}_{n}(\mathbf{x})=\mathbf{h}_{n}^{f}(\mathbf{x}),
$$

where $n$ is the channel number (corresponding to the number of parameters). Assuming that $F_{n i}$ is the $i$-th eigenvector of the $n$-th channel, $F_{n i}^{j}$ is the $j$-th position of the $i$ -th eigenvector of the $n$-th channel, and $I$ is the number of filters in the last layer, the classification contribution along the axis direction of the characteristic variable, $W_{V}^{c}$, satisfies the following conditions:

$$
\begin{aligned}
\beta_{n i}^{c} & =\frac{1}{t} \sum_{j=1}^{t} \frac{\partial S_{c}}{\partial F_{n i}^{j},} \\
W_{V}^{c} & =\frac{1}{t} \sum_{j} \operatorname{ReLU}\left(\sum_{i} \beta_{n i}^{c} F_{n i}^{j}\right) .
\end{aligned}
$$

3.3. Thermal Diagrams. Grad-CAM, in the form of equations (8) and (10), can be used to highlight the subsequences that contribute most to the classification process. It thus provides a visual interpretation of the data that can be used to accumulate experience and knowledge related to the classification process and make decisions using the model. However, this is only useful if the correct classification has been made. That is, only when the classification model is effective can the features related to the classification have practical significance. Three indices are therefore used to evaluate the accuracy of the classification process: the accuracy rate, $P$, the recall rate, $R$, and $F 1$, which is a comprehensive measure of the accuracy and recall rate:

$$
\begin{aligned}
P & =\frac{\mathrm{TP}}{\mathrm{TP}+\mathrm{FP}}, \\
R & =\frac{\mathrm{TP}}{\mathrm{TP}+\mathrm{FN}}, \\
F 1 & =\frac{2 \mathrm{PR}}{P+R},
\end{aligned}
$$

where TP is the number of true positive results, FP the number of false positive results, and FN the number of false negative results.

Provided the classification accuracy meets certain requirements, the classification contribution degrees along the time axis direction $\left(W_{T}^{c}\right)$ and characteristic variable direction $\left(W_{V}^{c}\right)$ are calculated. Then, these two results are unified, using F1 indices to weigh their relative contributions, to form the final classification contribution, $W_{V T}^{c}$, that is used to form the thermal diagram:

$$
W_{V T}^{c}=\operatorname{Norm}\left(W_{V}^{c}\right)+\operatorname{Norm}\left(W_{T}^{c}\right) * \frac{F 1_{T}}{F 1_{V}},
$$

where "Norm" represents normalization and $F 1_{T}$ and $F 1_{V}$ are the scores calculated using the ResNet and MCFCN, respectively. The classification contribution degrees represent the relative importance of the features to the classification process. Equation (12) implies that the classification contributions $W_{T}^{c}$ (along the time axis direction) and $W_{V}^{c}$ (along the parameter direction) are first normalized and then superimposed according to their $F 1$ values. Finally, a thermal diagram is obtained for the MTS based on the classification contributions $W_{V T}^{c}$. The calculation code of the visualization method shown in Figure 8 can be found in the appendix of this paper.

The thermal map of classification contributions provides an intuitive interpretation of the data that is especially useful when there is an absence of domain knowledge. It also provides a method for analyzing classification characteristics and accumulating classification experience. The training of the two network structures shown in Figure 8 is performed by minimizing the categorical cross-entropy. In this work, a balanced sampling strategy was used and an Adam optimization algorithm was employed to carrying out the minimization process. In addition, the strategies for learning rate adjustment are as follows:

$$
l r_{\text {new }}=l r_{\text {initial }} \times \gamma,
$$

where $l r_{\text {new }}$ is the updated learning rate, $l r_{\text {initial }}$ is the initial learning rate, and $\gamma$ is the multiplication factor of the updated learning rate. $\gamma=0.5$ is set in this paper, and the minimum learning rate is 0.0001 .

\section{Case Study}

4.1. Background Information. Ground loss during EPB shield tunneling is closely related to the prevailing geological conditions. Ground loss will eventually lead to surface settlement and is related to the depth of the tunnel. However, the effects arising due to geological conditions are complex. For example, tunnels excavated in sandy cobble areas may form arch structures above them which prevent the ground loss from being adequately reflected on the surface. Therefore, it is necessary to simplify matters by assuming that the geological conditions are ideal soft soil. Thus, we only study the loss occurring in soft soil and this must be reflected in our choice of test cases.

The cases considered in this paper all relate to EPB shield tunneling carried out in the Changzhou Metro area in Jiangsu, China. The landform in this area is best described as belonging to an alluvial lacustrine plain. The terrain is generally flat with some parts that are slightly undulating. The shallow parts just below the ground are mostly covered by late Pleistocene clay and silt. Here, we consider 13 EPB shield tunneling projects that mainly pass through silty sand and silty clay; the overlying soil layer is mainly miscellaneous 
fill and silty clay. The average burial depth of all the tunnel axes is $15.89 \mathrm{~m}$, which is typical for tunnel projects in urban soft soil areas. Based on the measured data of the geological survey, the five key parameters of compression modulus $E_{s}$, cohesion $c$ and internal friction angle $\varphi$, standard penetration modified blow count NSPT and density $\rho$ are calculated, respectively, as shown in Table 2.

The statistical results of each parameter are the average values of all survey points of the corresponding soil layer along each tunnel section, and the samples of each survey point are more than 3. By calculating the dispersion coefficient (the ratio of standard deviation to average) of each parameter, it can be seen that the dispersion coefficient of each parameter is less than $19.95 \%$. Therefore, the stratum conditions of 13 tunnel sections are similar and comparable, meeting the requirements of controlling geological conditions. A CTE6440 EPB shield machine was used in the construction of the 13 tunnels selected for analysis. The main parameters of this EPB shield machine and the tunnels constructed are shown in Table 3.

Our model for the EPB shield machine takes the construction of each supporting ring as the basic unit of measure and samples all parameters synchronously. The data sampling associated with each ring starts from the advance of the shield until the advance displacement meets that required for segment installation. The construction process used for each supporting segment is different, so the data length is not the same. However, the structure of the deep-learning model and parallel GPU computations performed on the time series require that all the time series have the same length. As shown in Figure 7, the MTS data for the ground loss due to the shield tunneling is obtained by splicing the continuous data recorded during the construction process. If the amount of data collected for each ring is excessive, the number of calculations will be large and this can affect the calculation accuracy. Conversely, if the amount of data is too little, information will be lost and this will also affect the accuracy of the results.

A statistical analysis shows that the minimum effective data length associated with 10,376 rings is 24 and the maximum value is 79 . Furthermore, the average value is 38 and the mode is 34 . Only $5.39 \%$ of the total number is more than 55. Therefore, in this paper, the data length for each ring is 55 , and less than 55 rings are filled with 0 . In addition, monitoring points to monitor the surface settlement were arranged every 5 rings along the direction of travel of the tunneling machine in the 13 tunnel projects. There are many factors that may affect the monitoring of surface settlement (e.g., presence of rivers, buildings, and other structures on the surface above the actual project) and the launching and receiving of the shield during the construction process can also be disturbed by many factors. Therefore, any surface settlement-monitoring point that was likely to have been adversely affected was removed from the analysis carried out in this paper, leaving a total of 1,822 effective monitoring points. The final surface settlements $\left(S_{\max }\right)$ at each point were obtained after waiting for at least 180 days after the corresponding support segments were installed.
4.2. Data Processing. Ground loss due to EPB shield tunneling is a dynamic and continuous process and the ground loss at a given surface settlement-monitoring point is usually related to the construction of several rings. Considering the engineering background outlined in Section 4.1, substitution of the shield construction parameters given in Table 3 into equations (3) and (4) yields the results $b=7$ and $a=5$. That is, the ground loss needs to be determined starting from ring number $S-7$ and ending at ring number $S+5$. Therefore, the ground loss due to the tunneling activity is related to the process of constructing 13 rings in the shield in total at each point. As indicated in Table 3, a total of 59 construction parameters are recorded by this model of EPB shield machine. This includes a great deal of important information that is fed back to the shield machine to control the tunneling process. The most relevant parameters, however, are determined according to the four main influencing factors mentioned in Section 2.2, as shown in Table 1.

It can be seen from Table 1 that $X_{N}$ has dimensions corresponding to $18 \times 715$. In theory, the ground loss at each effective settlement-monitoring point, together with the 13-ring data before and after it, constitutes the data sample $\left(X_{N}, Y_{N}\right)$. However, there is often some data that is found to be abnormal or missing during the actual construction process. Considering the influence that missing data has on the MTS model, only the monitoring points whose number of rings missing data is less than or equal to 1 are selected for use in this paper. Thus, a total of 1,182 settlement-monitoring points are finally deemed to be acceptable. As described in Section 2.1, the surface settlement $S_{\max }$ of each settlement-monitoring point is multiplied by the depth $H$ (from the corresponding tunnel axis to the ground) to obtain the ground loss $V_{\text {loss }}$. The calculated $V_{\text {loss }}$ values are distributed in the manner shown in Figure 9.

The 1,182 ground loss $V_{\text {loss }}$ values shown in Figure 9 approximately conform to a Cauchy distribution; the average loss is $-0.106 \times 10^{-3} \mathrm{~m}^{2}$. The corresponding average final settlement, $\bar{S}_{\max }=-6.67 \mathrm{~mm}$, lies in the range of formation loss values that is considered "normal." Engineering practice suggests that the data points in the dataset corresponding to $Y_{N}$ can be divided into three categories according to their average final settlement values $\bar{S}_{\max }$ (shown in Figure 9). The first type (class I) corresponds to ground losses $V_{\text {loss }}>-0.050 \times 10^{-3} \mathrm{~m}^{2}$, corresponding to $\bar{S}_{\max }>-3.146 \mathrm{~mm}$, which indicates that the subsidence is small with a small amount of loss or surface uplift. The second type (class II) is such that $-0.050 \times 10^{-3} \mathrm{~m}^{2} \geq$ $V_{\text {loss }}>-0.160 \times 10^{-3} \mathrm{~m}^{2}$ corresponding to $-3.146 \mathrm{~mm} \geq$ $\bar{S}_{\max }>-10.069 \mathrm{~mm}$, which indicates that there is a normal amount of formation loss and normal settlement. The third type (class III) corresponds to the condition $V_{\text {loss }} \leq-0.160 \times$ $10^{-3} \mathrm{~m}^{2}$ corresponding to $\bar{S}_{\max } \leq-10.069 \mathrm{~mm}$, which indicates that excessive ground loss and settlement is occurring.

The 1,182 labeled samples were divided into three categories according to the above classification method. Each parameter was normalized to make the largest value equal to one (all the data was normalized apart from those entries filled with zeros). The data points were then randomly 
TABLE 2: Statistical table of main geological parameters of 13 shield tunnel projects.

\begin{tabular}{|c|c|c|c|c|c|}
\hline Shield tunneling projects & $\rho\left(\mathrm{g} / \mathrm{cm}^{3}\right)$ & $E_{\mathrm{s}}(\mathrm{MPa})$ & $c(\mathrm{kPa})$ & $\varphi\left(^{\circ}\right)$ & $N_{\mathrm{SPT}}$ \\
\hline 1 & 1.89 & 9.94 & 7.00 & 30.67 & 30.37 \\
\hline 2 & 1.91 & 11.11 & 5.00 & 30.44 & 24.52 \\
\hline 3 & 1.97 & 10.79 & 8.68 & 30.83 & 24.22 \\
\hline 4 & 1.93 & 12.5 & 5.08 & 32.25 & 30.31 \\
\hline 5 & 1.93 & 12.5 & 5.08 & 32.26 & 30.33 \\
\hline 6 & 1.92 & 11.26 & 5.92 & 31.6 & 29.23 \\
\hline 7 & 1.91 & 11.26 & 5.94 & 31.61 & 29.24 \\
\hline 8 & 1.93 & 9.98 & & 32.47 & 23.22 \\
\hline 9 & 1.93 & 9.98 & & 32.47 & 23.22 \\
\hline 10 & 1.96 & 11.03 & 8.40 & 31.16 & 23.5 \\
\hline 11 & 1.94 & 10.35 & 6.57 & 22.27 & 23.57 \\
\hline 12 & 1.94 & 10.35 & 6.58 & 22.31 & 23.53 \\
\hline 13 & 1.95 & 12.11 & 5.00 & 31.76 & 27.98 \\
\hline Dispersion coefficient & 1.07 & 7.95 & 19.95 & 11.33 & 11.42 \\
\hline
\end{tabular}

TABLE 3: Key characteristics of the tunnels/tunneling machine considered in this work.

\begin{tabular}{lc}
\hline Parameter & Value \\
\hline Length of the EPB shield machine, $L_{m}(\mathrm{~m})$ & $9.10 \sim 9.40$ \\
Distance between the panel and supporting segment under construction, $L_{s}(\mathrm{~m})$ & $5.90 \sim 6.10$ \\
Width of supporting segment, $l(\mathrm{~m})$ & 1.20 \\
Outer diameter of shield machine, $D(\mathrm{~m})$ & $6.34 \sim 6.38$ \\
Frequency with which the machine parameters are sampled $(\mathrm{Hz})$ & 0.02 \\
Total length of tunnels $(\mathrm{m})$ & 12,451 \\
Total number of rings used to support the tunnels & 10,376 \\
Number of parameters recorded by the shield machine & 59 \\
\hline
\end{tabular}

divided into a training set and a verification set (in a ratio of $4: 1$, so that the verification set consisted of 236 data points). This produced the final MTS model, $D T=\left\{\left(X_{1}, Y_{1}\right),\left(X_{2}, Y_{2}\right), \ldots,\left(X_{N}, Y_{N}\right)\right\}$, used to model the ground loss caused by the EPB shield tunneling carried out in this region.

4.3. Results and Discussion. The number of rings used to correlate with ground loss is one of the key parameters of the MTS model. In addition, the MTS model is based on a new dataset and so the effectiveness of the algorithms used needs to be verified by comparison with existing methods.

4.3.1. Model Validation. Using equations (3) and (4), it was calculated that the starting ring to use should be $S-7$ and the ending ring should be $S+5$ when calculating the ground loss (that is, the number of rings that are relevant to the ground loss due to EPB shield tunneling is 13). In order to verify this, the results obtained using a variety of values for $a$ and $b$ are compared keeping all other parameters the same. The ResNet method shown in Figure 8 is used to perform the calculations, producing the results shown in Table 4 .

Table 4 shows that the classification accuracy tends to increase as the total number of correlation rings is increased. Clearly, using just one ring produces the worst results. It also shows that the ground loss caused by shield tunneling is related to the construction of multiple rings. The classification accuracy, however, does not increase linearly with the number of correlation rings considered. This suggests that correlation between the ground loss correlation rings also affects the results. The most important aspect of Table 4, however, is that of all the working conditions used, the best classification effect was achieved by using rings $S-7$ to $S+5$. This supports the method used here to calculate which rings are the most appropriate ones to use to calculate the ground loss, i.e., those calculated according to equations (3) and (4).

4.3.2. Algorithm Comparison. The new MTS model has its own characteristics and needs to be checked for accuracy. Methods based on ResNets and FCNs tend to have the best classification accuracy and stability according to their performance with existing MTS datasets. In order to construct classification contribution thermal diagrams, in this paper, we proposed using an MCFCN method (referring to a combination of an MCDCNN and an FCN). In addition, other people have used an MLSTM-FCN method [51], which combines an LSTM with an FCN and classifies the MTS into unitary time series (providing an interface for the latter [52]). These different MTS classification methods are applied to the current MTS model, $D T=\left\{\left(X_{1}, Y_{1}\right),\left(X_{2}, Y_{2}\right)\right.$, $\left.\ldots,\left(X_{N}, Y_{N}\right)\right\}$, for the ground loss due to EPB shield tunneling. The results are shown and compared in Figure 10 (where 10 repetitions are performed using each method).

Figure 10 shows that the ResNet method has the best classification accuracy and stability among the different MTS classification methods, producing the best $F 1$ score of 0.854 . 


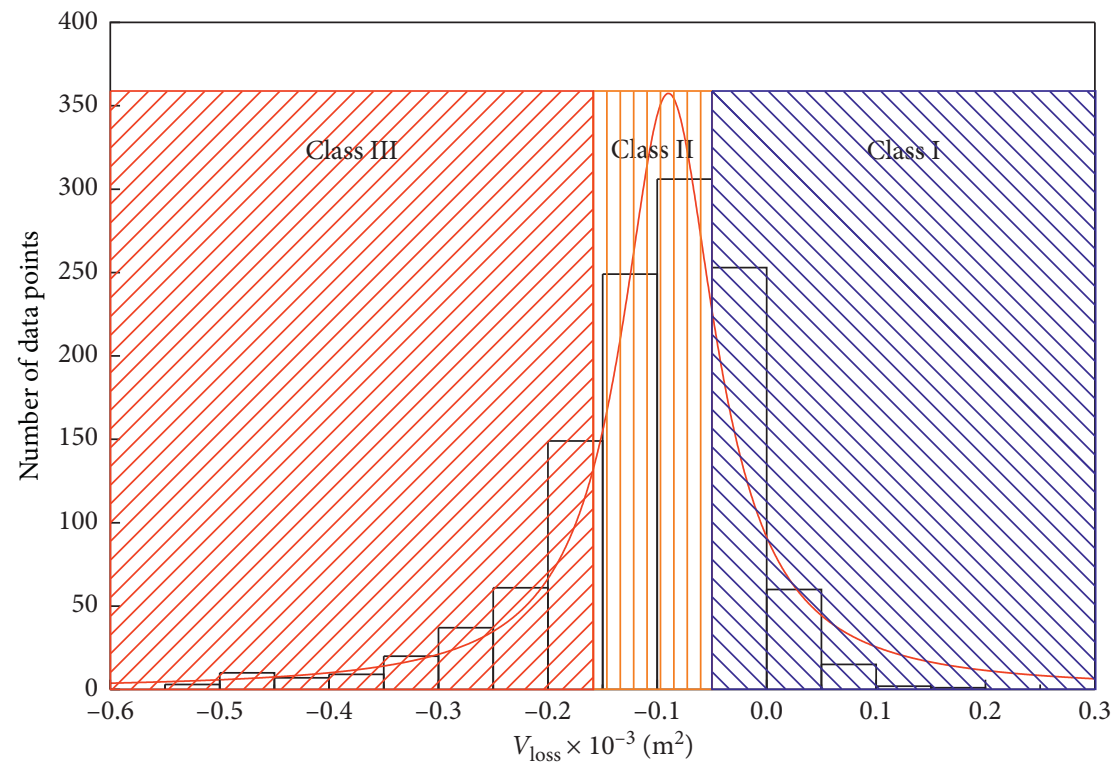

Frequency histogram of ground loss $V_{\text {loss }}$

Figure 9: Statistical distribution of the ground loss values, $V_{\text {loss. }}$.

TABLE 4: Comparison of the effect of changing the number of rings used in the correlation.

\begin{tabular}{lcccccc}
\hline Working conditions & $a$ & $b$ & Total & Precision & Recall & $F 1$ \\
\hline 1 & 0 & 0 & 1 & 0.624 & 0.614 & 0.619 \\
2 & 1 & 1 & 3 & 0.696 & 0.720 & 0.708 \\
3 & 2 & 2 & 5 & 0.689 & 0.695 & 0.692 \\
4 & 3 & 3 & 7 & 0.719 & 0.684 & 0.701 \\
5 & 4 & 4 & 9 & 0.790 & 0.788 & 0.789 \\
6 & 5 & 5 & 11 & 0.792 & 0.806 & 0.799 \\
7 & 6 & 6 & 13 & 0.788 & 0.815 & 0.801 \\
$\mathbf{8}$ & $\mathbf{5}$ & $\mathbf{7}$ & $\mathbf{1 3}$ & $\mathbf{0 . 8 5 7}$ & $\mathbf{0 . 8 5 2}$ & $\mathbf{0 . 8 5 4}$ \\
9 & 7 & 7 & 15 & 0.758 & 0.748 & 0.753 \\
10 & 8 & 8 & 17 & 0.766 & 0.754 & 0.760 \\
11 & 9 & 9 & 19 & 0.810 & 0.802 & 0.806
\end{tabular}

Bold values represent the best working condition.

The FCN method also produced good results, although its average accuracy $(F 1=0.842)$ and stability are lower than the ResNet method. In addition, although the MLSTM-FCN method combines an FCN with an LSTM, the classification effect is not outstanding. In the multichannel multivariate time classification method, the MCFCN method proposed in this paper is clearly better than using an MCDCNN method. Therefore, as shown in Figure 8, the ResNet and MCFCN methods can effectively classify the MTS ground loss data and ensure the reliability of the relative weights of the classification contributions.

4.3.3. Visualization of MTS Features. The ResNet and MCFCN methods are used to classify the MTS model for the ground loss and calculate the various $F 1$ scores, giving the results shown in Table 5. The calculation data and code of this case can be referred to the appendix of this paper.

Thermal diagrams can now be drawn to give a visual representation of the contributions made by the various

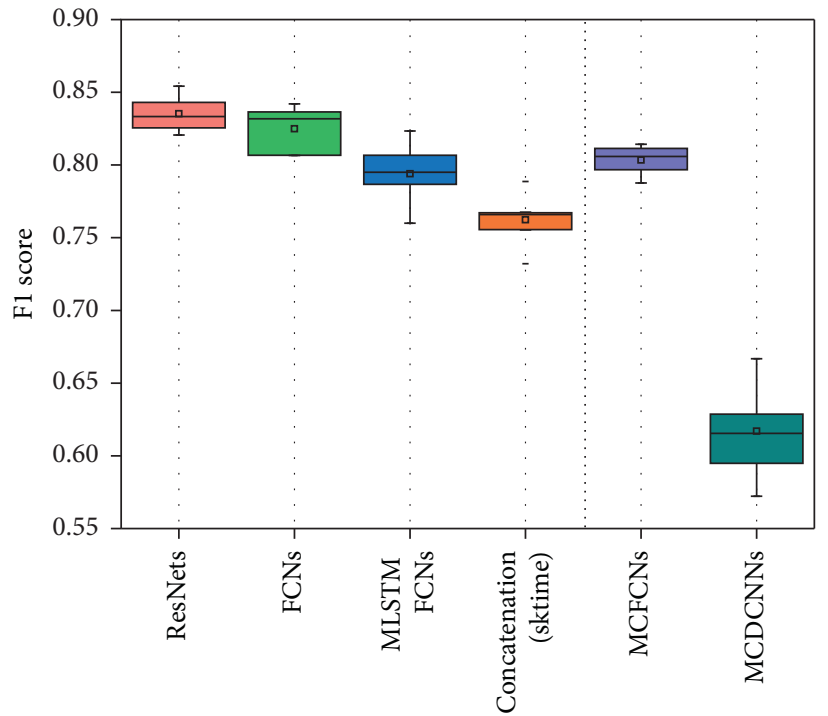

Figure 10: Comparison of results obtained using different MTS classification algorithms.

TABLe 5: F1 scores.

\begin{tabular}{lccc}
\hline Class & $F 1_{T}$ & $F 1_{V}$ & $F 1-$ mean \\
\hline I & 0.809 & 0.832 & 0.821 \\
II & 0.857 & 0.829 & 0.843 \\
III & 0.896 & 0.772 & 0.834 \\
\hline
\end{tabular}

rings and parameters to the ground loss data for each of the classes (Figure 11). The abscissae in Figure 11 indicate which ring is being considered (where $S$ is the ring number of the ground settlement-monitoring point). The ordinates indicate which parameter is involved, the serial numbers corresponding to those in Table 1 . The color represents the 


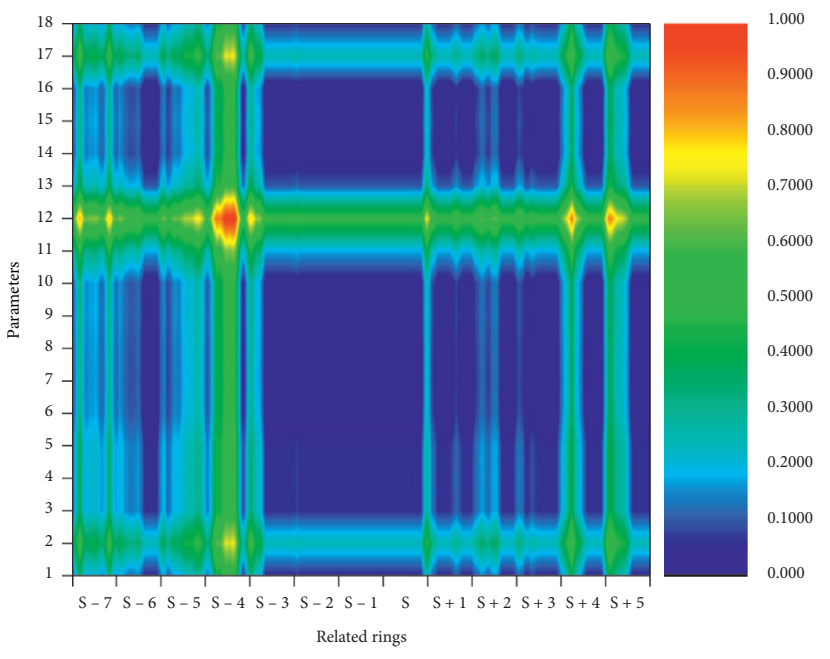

(a)

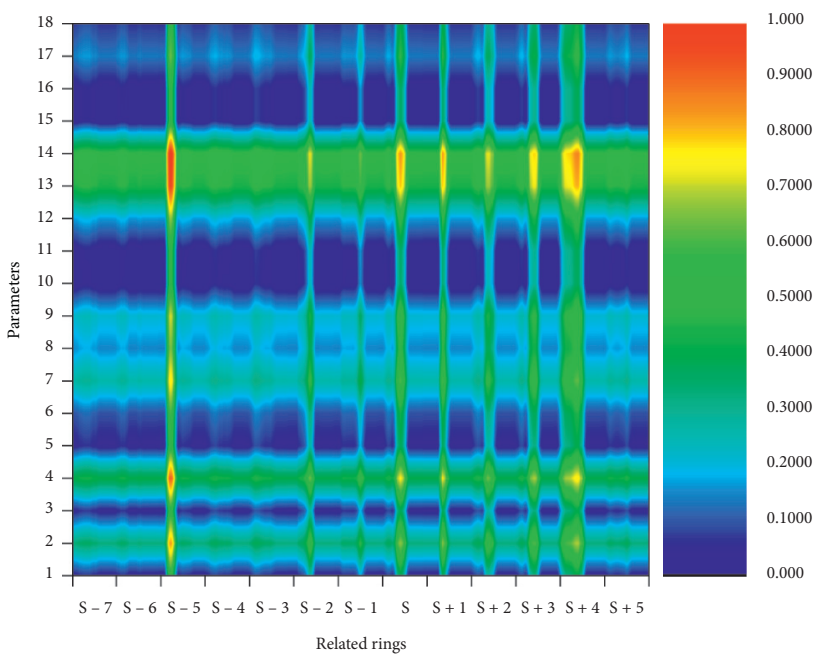

(b)

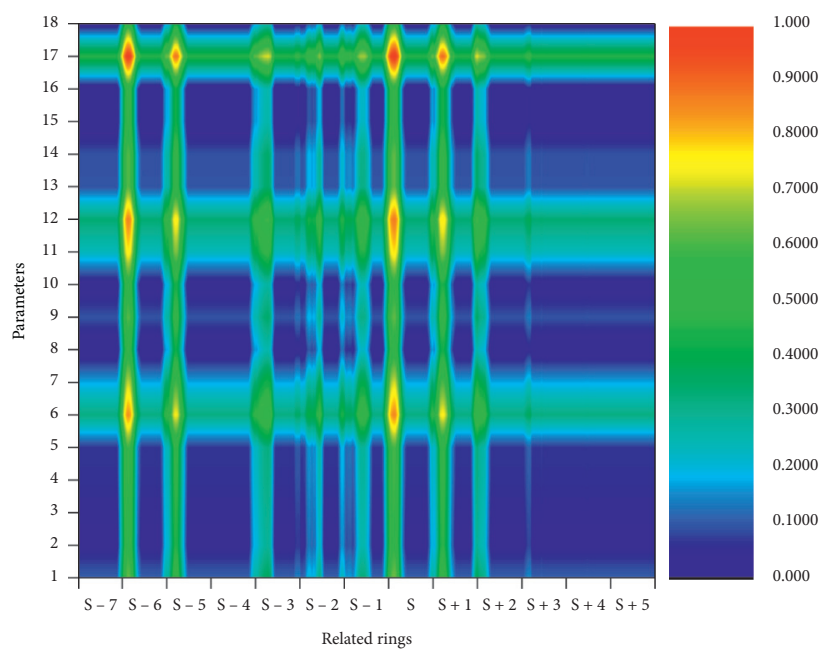

(c)

Figure 11: Thermal diagrams showing the contributions made to the different classes. (a) Class I. (b) Class II. (c) Class III.

relative weight of the classification as calculated using equation (12).

Figure 11 shows that there are significant differences between the thermal diagrams calculated for the three classes. The key parameters to judge the data points in class I are the average articulation displacement, the total amount of grouting, and the thrust pressure-down. In addition, it is the ring $S-4$ along the time direction that is the most important one to judge class I. Similar observations can be made about the other classes. The key parameters to judge class II are the horizontal difference between the front and rear points, the vertical difference between the front and back points, the thrust pressure-down and thrust pressureright (related to the control of the shield's posture), and the rings $S-5$ and $S+4$ along the time direction. Finally, the key parameters to judge class III are the total amount of grouting, the earth chamber pressure-medium, the average advance displacement, and the average articulation displacement. In this case, rings $S-6$ and $S$ are the most important rings for judging class III along the time direction.
As already mentioned, the higher the $F 1$ score (Table 5), the more reliable the contribution to the thermal classification map. Therefore, such maps not only provide a visual method of interpreting the classified MTS data but also provide a means of further analyzing the classification of the related features.

In practice, it is the third kind of ground settlement (class III) that is of most concern as the excessive amount of ground loss involved can cause damage. Considering the form of the thermal map shown in Figure 11(c), we take the parameter corresponding to earth chamber pressure-medium (index number 6) as an example and analyze the distribution of the relative weights along the time direction in more detail, producing the results shown in Figure 12. In this figure, the color represents the relative weight of the classification, as calculated using equation (8) and represents a basis with which class III can be judged.

As shown in Figure 12, the relative weights are mainly focused on the data related to the effective construction process, and the parts filled with zeros after each ring data 


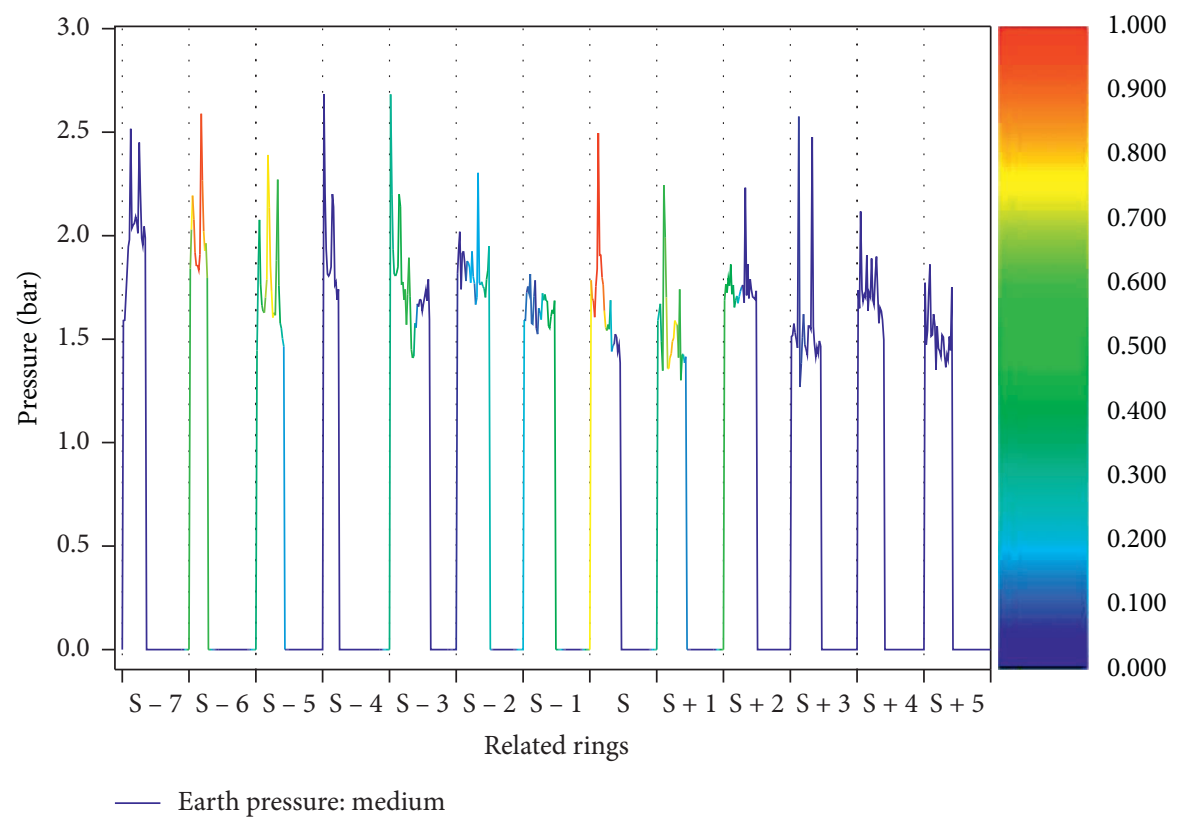

FIGURE 12: Distribution of the relative weights in class III along the construction direction.

are almost ignored. From the perspective of the distribution of relative weight of classification, rings $S-6$ and $S$ are the most important for judging class III, followed by rings $S-5$ and $S+1$, which provides a visual method of analyzing features. Clearly, when identifying the risk of excessive ground loss corresponding to class III, attention should be paid to these four rings. The enlarged parts shown in Figure 12 indicate that the change in the relative weights is continuous, and the parts with high weights are similar to the key local features of the time series, which can therefore accumulate experience for further analysis and extraction of relevant features.

\section{Conclusions}

Ground loss as a result of EPB shield tunneling is a dynamic and continuous process. This paper puts forward an MTS model for ground loss based on an analysis of the most relevant factors and processes associated with ground loss combined with the characteristics of the time series data for various parameters recorded by the shield machine in real time. An MTS feature visualization method based on a ResNet and MCFCN is also presented. The following conclusions can be drawn:

(1) The MTS model proposed in this paper is effective and produced an $F 1$ score of 0.854 based on a ResNet classification method.

(2) The ResNet method produced the best and most stable classification performance when applied to the MTS model for ground loss. The MCFCN constructed in this paper also performed significantly better than the existing MCDCNN methods.

(3) The method used in this paper to visualize the features of the MTS classification results allows the contributions to the classifications to be presented using thermal maps. It thus becomes easy to identify the key parameters and important parts that make the largest contributions to the classification process. The method not only provides good interpretability for the classification of the MTS but also provides a visual basis for further analyzing the most relevant features.

In addition, the MTS feature visualization method of ground loss caused by EPB shield tunneling is based on the classification effect. The better the classification effect is, the higher the credibility of the features obtained by the method is. Therefore, the future work is to improve the classification effect and apply the method to more engineering cases. Through analyzing the characteristics of a large number of data and summarizing the experience and knowledge of classification, the effective judgment of ground loss is realized, which lays the foundation for the effective control of ground loss.

\section{Data Availability}

The codes and data files used to support the findings of this study are available from the corresponding author upon request.

\section{Conflicts of Interest}

The authors declare no conflicts of interest.

\section{Authors' Contributions}

Zhen Wang conceived, designed, and performed the study. Xiaoli Rong and Dong An collected and analyzed the example used in the paper. Zhu Wen and Fei Gao wrote and 
revised the paper together. The authors have read and approved the final published manuscript.

\section{Acknowledgments}

This work was supported by the National Natural Science Foundation of China (42002266, 51909120, and 12002171).

\section{References}

[1] R. B. Peck, "Deep excavation and tunneling in soft ground," in Proceedings of the International Conference in Soil Mechanics and Foundation Engineering, pp. 225-290, January 1969, https://www.issmge.org/uploads/publications/1/38/1969_03_ 0010.pdf.

[2] S. R. Dindarloo and E. S. Irdemoosa, "Maximum surface settlement based classification of shallow tunnels in soft ground," Tunnelling and Underground Space Technology, vol. 49, pp. 320-327, 2015.

[3] Y. Fang, C. He, A. Nazem, Z. Yao, and J. Grasmick, "Surface settlement prediction for EPB shield tunneling in sandy ground," KSCE Journal of Civil Engineering, vol. 21, no. 7, pp. 2908-2918, 2017.

[4] C. Sagaseta, "Discussion: analysis of undrained soil deformation due to ground loss," Géotechnique, vol. 38, no. 4, pp. $647-649,1988$.

[5] A. Bobet, "Analytical solutions for shallow tunnels in saturated ground," Journal of Engineering Mechanics, vol. 127, no. 12, pp. 1258-1266, 2001.

[6] X. Liu, Q. Fang, D. Zhang, and Y. Liu, "Energy-based prediction of volume loss ratio and plastic zone dimension of shallow tunneling," Computers and Geotechnics, vol. 118, 2020.

[7] K. M. Lee and R. K. Rowe, "Finite element modelling of the three-dimensional ground deformations due to tunnelling in soft cohesive soils: part I - method of analysis," Computers and Geotechnics, vol. 10, no. 2, pp. 87-109, 1990.

[8] K. M. Lee, R. K. Rowe, and K. Y. Lo, "Subsidence owing to tunnelling. I. estimating the gap parameter," Canadian Geotechnical Journal, vol. 29, no. 6, pp. 929-940, 1992.

[9] S. R. Moeinossadat and K. Ahangari, "Estimating maximum surface settlement due to EPBM tunneling by NumericalIntelligent approach - a case study: Tehran subway line 7," Transportation Geotechnics, vol. 18, pp. 92-102, 2019.

[10] S. Miliziano and A. D. Lillis, "Predicted and observed settlements induced by the mechanized tunnel excavation of metro line C near S. Giovanni station in Rome," Tunnelling and Underground Space Technology, vol. 86, pp. 236-246, 2019.

[11] N. V. Minh, B. Wout, and B. Johan, "Volume loss in shallow tunneling," Tunnelling and Underground Space Technology, vol. 59, pp. 77-90, 2016.

[12] F. Y. Meng, R. P. Chen, and X. Kang, "Effects of tunnelinginduced soil disturbance on the post-construction settlement in structured soft soils," Tunnelling and Underground Space Technology, vol. 80, pp. 53-63, 2018.

[13] Z. Wang, W. Yao, Y. Cai, B. Xu, Y. Fu, and G. Wei, "Analysis of ground surface settlement induced by the construction of a large-diameter shallow-buried twin-tunnel in soft ground," Tunnelling and Underground Space Technology, vol. 83, pp. 520-532, 2019.

[14] C. Zhou, L. Y. Ding, M. J. Skibniewski, H. Luo, and H. T. Zhang, "Data based complex network modeling and analysis of shield tunneling performance in metro construction," Advanced Engineering Informatics, vol. 38, pp. 168-186, 2018.

[15] D. Festa, W. Broere, and J. W. Bosch, "An investigation into the forces acting on a TBM during driving - mining the TBM logged data," Tunnelling and Underground Space Technology, vol. 32, pp. 143-157, 2012.

[16] C. Y. Kim, G. J. Bae, S. W. Hong, C. H. Park, H. K. Moon, and H. S. Shin, "Neural network based prediction of ground surface settlements due to tunnelling," Computers and Geotechnics, vol. 28, no. 6-7, pp. 517-547, 2001.

[17] S. Suwansawat and H. H. Einstein, "Artificial neural networks for predicting the maximum surface settlement caused by EPB shield tunneling," Tunnelling and Underground Space Technology, vol. 21, no. 2, pp. 133-150, 2006.

[18] A. Darabi, K. Ahangari, A. Noorzad, and A. Arab, "Subsidence estimation utilizing various approaches-a case study: Tehran No. 3 subway line," Tunnelling and Underground Space Technology, vol. 31, pp. 117-127, 2012.

[19] J. Ninić and G. Meschke, "Model update and real-time steering of tunnel boring machines using simulation-based meta models," Tunnelling and Underground Space Technology, vol. 45, pp. 138-152, 2015.

[20] P. Zhang, H.-N. Wu, R.-P. Chen, and T. H. T. Chan, "Hybrid meta-heuristic and machine learning algorithms for tunneling-induced settlement prediction: a comparative study," Tunnelling and Underground Space Technology, vol. 99, Article ID 103383, 2020.

[21] P. Zhang, R. Chen, and H. N. Wu, "Real-time analysis and regulation of EPM shield steering using random forest," Automation in Construction, vol. 106, 2019.

[22] P. Zhang, H. Li, Q. P. Ha, Z.-Y. Yin, and R. P. Chen, "Reinforcement learning based optimizer for improvement of predicting tunneling-induced ground responses," Advanced Engineering Informatics, vol. 45, Article ID 101097, 2020.

[23] F. Wang, B. Gou, and Y. Qin, "Modeling tunneling-induced ground surface settlement development using a wavelet smooth relevance vector machine," Computers and Geotechnics, vol. 54, pp. 125-132, 2013.

[24] D. Bouayad and F. Emeriault, "Modeling the relationship between ground surface settlements induced by shield tunneling and the operational and geological parameters based on the hybrid PCA/ANFIS method," Tunnelling and Underground Space Technology, vol. 68, pp. 142-152, 2017.

[25] K. Ahangari, S. R. Moeinossadat, and D. Behnia, "Estimation of tunnelling-induced settlement by modern intelligent methods," Soils and Foundations, vol. 55, no. 4, pp. 737-748, 2015.

[26] L. Zhang, X. Wu, W. Ji, and S. M. AbouRizk, "Intelligent approach to estimation of tunnel-induced ground settlement using wavelet packet and support vector machines," Journal of Computing in Civil Engineering, vol. 31, no. 2, Article ID 4016053, 2017.

[27] R. P. Chen, P. Zhang, X. Kang, Z. Q. Zhong, Y. Liu, and H. N. Wu, "Prediction of maximum surface settlement caused by earth pressure balance (EPB) shield tunneling with ANN methods," Soils and Foundations, vol. 59, no. 2, pp. 284-295, 2019.

[28] F. J. Ordóñez and D. Roggen, “Deep convolutional and LSTM recurrent neural networks for multi- modal wearable activity recognition," Sensors, vol. 16, no. 1, p. 115, 2016.

[29] H. F. Nweke, Y. W. Teh, M. A. Al-garadi, and U. R. Alo, "Deep learning algorithms for human activity recognition using mobile and wearable sensor networks: state of the art and 
research challenges," Expert Systems with Applications, vol. 105, pp. 233-261, 2018.

[30] Z. Wang, W. Yan, and T. Oates, "Time series classification from scratch with deep neural networks: a strong baseline," in Proceedings of the 2017 International Joint Conference on Neural Networks, pp. 1578-1585, Anchorage, Alaska, USA, May 2017.

[31] H. Ismail Fawaz, G. Forestier, J. Weber, L. Idoumghar, and P.-A. Muller, "Deep learning for time series classification: a review," Data Mining and Knowledge Discovery, vol. 33, no. 4, pp. 917-963, 2019.

[32] J. Wu, L. Yao, and B. Liu, "An overview on feature-based classification algorithms for multivariate time series," in Proceedings of the 2018 IEEE 3rd International Conference on Cloud Computing and Big Data Analysis (ICCCBDA), April 2008.

[33] B. Zhou, A. Khosla, A. Lapedriza, A. Oliva, and A. Torralba, "Learning deep features for discriminative localization," in Proceedings of the IEEE Conference on Computer Vision and Pattern Recognition, pp. 2921-2929, Las Vegas, NV, USA, June 2016.

[34] R. R. Selvaraju, M. Cogswell, A. Das, R. Vedantam, and D. Batra, "Grad-CAM: visual explanations from deep networks via gradient-based localization," International Journal of Computer Vision, vol. 128, no. 8, pp. 336-359, 2019.

[35] Y. Zheng, Q. Liu, E. Chen, Y. Ge, and J. L. Zhao, “Time series classification using multi-channels deep convolutional neural networks," in Proceedings of the International Conference on Web-Age Information Management, pp. 298-310, Macau, China, June 2014.

[36] Y. Zheng, Q. Liu, E. Chen, Y. Ge, and J. L. Zhao, "Exploiting multi-channels deep convolutional neural networks for multivariate time series classification," Frontiers of Computer Science, vol. 10, no. 1, pp. 96-112, 2016.

[37] P. Chambon and J. F. Corté, "Shallow tunnels in cohesionless soil: stability of tunnel face," Journal of Geotechnical Engineering, vol. 120, no. 7, pp. 1148-1165, 1994.

[38] P. Li, F. Wang, C. Zhang, and Z. Li, "Face stability analysis of a shallow tunnel in the saturated and multilayered soils in short-term condition," Computers and Geotechnics, vol. 107, pp. 25-35, 2018.

[39] C. Zhang, K. Han, and D. Zhang, "Face stability analysis of shallow circular tunnels in cohesive-frictional soils," Tunnelling and Underground Space Technology, vol. 50, pp. 345357, 2015.

[40] H. Shi, H. Yang, G. Gong, and L. Wang, "Determination of the cutterhead torque for EPB shield tunneling machine," Automation in Construction, vol. 20, no. 8, pp. 1087-1095, 2011.

[41] X. Liu, C. Shao, H. Ma, and R. Liu, "Optimal earth pressure balance control for shield tunneling based on LS-SVM and PSO," Automation in Construction, vol. 20, no. 4, pp. 321-327, 2011.

[42] A. S. Merritt and R. J. Mair, "Mechanics of tunnelling machine screw conveyors: a theoretical model," Géotechnique, vol. 58, no. 2, pp. 79-94, 2008.

[43] A. S. Merritt and R. J. Mair, "Mechanics of tunnelling machine screw conveyors: model tests," Géotechnique, vol. 56, no. 9, pp. 605-615, 2006.

[44] D. Festa, W. Broere, and J. W. Bosch, "Tunnelling in soft soil: tunnel boring machine operation and soil response," in Proceedings of the TU-Seoul 2013 Tunnelling and Underground Space Construction for Sustainable Development, Seoul, Korea, March 2013, https://www.researchgate.net/publication/
262012237_Tunnelling_in_Soft_Soil_Tunnel_Boring_ Machine_Operation_and_Soil_Response.

[45] D. Festa, W. Broere, and J. W. Bosch, "Kinematic behaviour of a tunnel boring machine in soft soil: theory and observations," Tunnelling and Underground Space Technology, vol. 49, pp. 208-217, 2015.

[46] M. Sharghi, H. Chakeri, and Y. Ozcelik, "Investigation into the effects of two component grout properties on surface settlements," Tunnelling and Underground Space Technology, vol. 63, pp. 205-216, 2017.

[47] A. Jallow, C. Y. Ou, and A. Lim, "Three-dimensional numerical study of long-term settlement induced in shield tunneling," Tunnelling and Underground Space Technology, vol. 88, pp. 221-236, 2019.

[48] J. Lines, S. Taylor, and A. Bagnall, "Time series classification with HIVE-COTE," ACM Transactions on Knowledge Discovery from Data, vol. 12, no. 5, pp. 1-35, 2018.

[49] S. Ioffe and C. Szegedy, "Batch normalization: accelerating deep network training by reducing internal covariate shift," in Proceedings of the 32nd International Conference on Machine Learning, pp. 448-456, Lille, France, July 2015.

[50] K. He, X. Zhang, S. Ren, and J. Sun, "Deep residual learning for image recognition," in Proceedings of the IEEE Conference on Computer Vision and Pattern Recognition, pp. 770-778, Seattle, WA, USA, July 2016.

[51] F. Karim, S. Majumdar, H. Darabi, and S. Harford, "Multivariate LSTM-FCNs for time series classification," Neural Networks, vol. 116, pp. 237-245, 2019.

[52] L. Markus and A. Bagnall, S. Ganesh, K. Viktor, J. Lines, and J. K. Franz, Sktime: a unified interface for machine learning with time series," 2019, https://arxiv.org/abs/1909.07872. 